\title{
Dormancy in Plants
}

Wim JJ Soppe, Max Planck Institute for Plant Breeding Research, Cologne, Germany

Leónie Bentsink, Wageningen University, Wageningen, The Netherlands

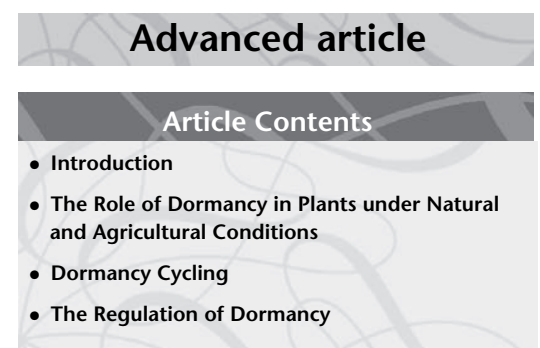

Online posting date: $16^{\text {th }}$ August 2016

\begin{abstract}
Dormancy is a strategy of higher plants to survive adverse conditions by pausing growth and development, which can occur in different organs like seeds and buds. Dormancy is controlled both by genetic and environmental factors and most of our knowledge about its regulation has been obtained by studying seeds. Seed dormancy is an important adaptive trait in wild plants and has been under negative selection during domestication. The plant hormone abscisic acid has been shown to play a crucial role in the establishment and maintenance of dormancy, whereas gibberellins promote germination. Additional regulators include reactive oxygen species and epigenetic modifications. Large differences in seed dormancy have been found within species and the identification of the underlying genes revealed several novel genes that specifically regulate dormancy.
\end{abstract}

\section{Introduction}

Dormancy is defined as 'the state of being not active or growing at the present time but able to be active later on' (Collins COBUILD dictionary). This is an important trait for plants that enables them to survive periods that are adverse for growth like drought, cold or heat. The development of dormancy during evolution enabled plants to inhabit environments that would be lethal for continuously growing plants without a dormant phase. This plant feature can manifest itself in different organs like seeds, buds, tubers and bulbs (Figure 1). During the dormant state, metabolic activity is nearly absent and development comes to a standstill. Dormancy is often associated with low moisture levels and protective structures like a seed coat in seeds or protective scales in buds and bulbs. Induction usually takes place towards the end of a growth season and release at the beginning of the next one. Dormancy has

eLS subject area: Plant Science

How to cite:

Soppe, Wim JJ and Bentsink, Leónie (August 2016) Dormancy

in Plants. In: eLS. John Wiley \& Sons, Ltd: Chichester.

DOI: 10.1002/9780470015902.a0002045.pub2 been studied in different plant organs with a focus on seed and bud dormancy. Seeds have the advantage of being independent entities which can be studied in rapidly cycling annual plants, in contrast to buds that are often part of slow-cycling perennial plants. Consequently, most progress in our understanding has been achieved using seeds and this article will therefore focus on seed dormancy. See also: Seeds

\section{The Role of Dormancy in Plants under Natural and Agricultural Conditions}

The moment when a seed germinates determines the conditions that it will encounter as a seedling and adult plant. Germination timing is regulated by seed dormancy, which has a major role in the adaptation of plants to their environment. Similarly, the appropriate timing of bud dormancy release is important to ensure bud flush at the beginning of the growth season. See also: Local Adaptation in Plants

Dormancy levels vary between plant species. In general, the number of plant species that may acquire dormancy shows a trend to increase with geographical distance from the equator and correlates with the occurrence of seasons (Baskin and Baskin, 2014). Variation can also be found within plant species. This has been studied intensively in the model species Arabidopsis thaliana (Arabidopsis), which is widespread in Europe and parts of Asia and shows high variation in dormancy levels among accessions (Figure 2). Accessions from southern Europe show a tendency for higher seed dormancy levels compared to accessions from northern Europe (Debieu et al., 2013). This could be an adaptation to the dry summers in the south. However, a strong correlation between latitude and dormancy level has not been found and accessions are probably adapted to specific local conditions. The genetic variation has been studied by crossing genotypes with different dormancy levels in species like Arabidopsis and rice, followed by analysis of their progeny (Alonso-Blanco et al., 2003; Lin et al., 1998). This has led to the identification of quantitative trait loci (QTL) and subsequent identification of some of the underlying genes like DELAY OF GERMINATION 1 (DOG1) in Arabidopsis (Bentsink et al., 2006) and Seed Dormancy 4 (SDR4) in rice (Sugimoto et al., 2010). These genes encode specific regulators of dormancy that are further discussed below. See also: Natural Variation as a Tool for Gene Identification in Plants 


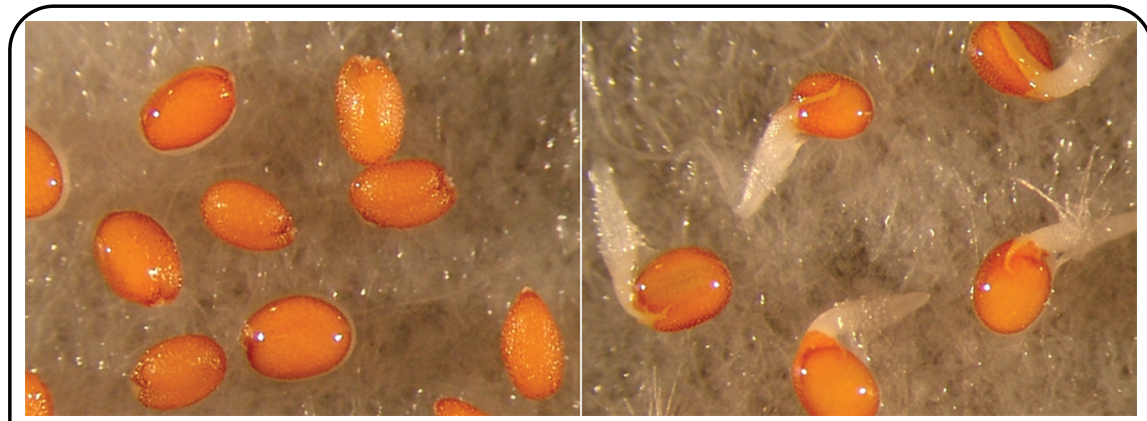

(a)

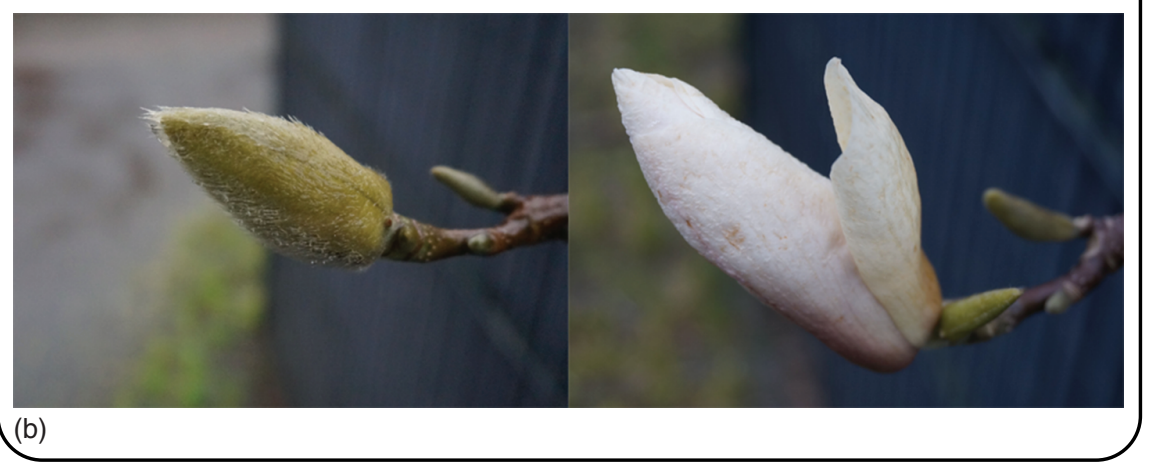

Figure 1 Dormancy in plants. (a) Seed dormancy in Arabidopsis. The photos show dormant (left) and fully after-ripened (right) seeds that were imbibed in the light for 3 days. (b) Bud dormancy in Magnolia. The photos show the same bud in a dormant (left) and nondormant (right) state. The photo on the left was taken on 3 February 2016 and the photo on the right on 30 March 2016 in Dansweiler, Germany.

Plants that are adapted to a specific location can still experience diverse environmental conditions from year to year. Therefore, seeds are able to sense environmental factors, like temperature, in order to release their dormancy at the right moment. It has, for instance, been demonstrated that the temperature that the mother plant experiences during seed maturation has a strong influence, with lower temperatures leading to more intensive seed dormancy (Kendall and Penfield, 2012). The adaptive nature of dormancy can also give plants some flexibility to set the timing of seed germination and bud flush to adapt to climate change.

Seeds of crop plants do not need to adapt to their environment for germination timing because this is taken care of by the farmer who sows the seeds at the appropriate moment at the beginning of the growth season. These seeds should germinate rapidly and uniformly for fast crop establishment, leading to high yield. Consequently, seed dormancy is a disadvantage for most crops and this trait has been largely lost during domestication. However, too low levels can be a disadvantage and can lead to reduced seed quality and pre-harvest sprouting (Figure 3), especially in cereals (Rodríguez et al., 2015). Therefore, seeds of most crops have an optimal level of dormancy and its control is an important goal in breeding programs.

\section{Dormancy Cycling}

Freshly matured seeds often contain primary dormancy, which is induced during seed development on the mother plant and allows delay of germination until the start of the growing season (Hilhorst, 1998; Finch-Savage and Leubner-Metzger, 2006). Seeds that are not exposed to favourable conditions for germination will not germinate and can enter the seed soil bank and acquire secondary dormancy (Hilhorst, 1998; Finch-Savage and Leubner-Metzger, 2006; Baskin and Baskin, 2014). Secondary dormancy can be induced and relieved in the same seed repeatedly for several cycles, until the required germination conditions appear. Like primary dormancy, secondary dormancy is affected by genetic and environmental factors. Although little is known about how it is regulated, similar genes might be involved as in primary dormancy. For example, the expression of DOG1, which was first identified to regulate primary dormancy (Bentsink et al., 2006), is controlled by environmental signals (mainly temperature) during dormancy cycling (Footitt et al., 2011). Likely, DOG1 is important for indicating the correct season for germination.

\section{The Regulation of Dormancy}

\section{Role of plant hormones in the control of seed dormancy}

Since several decades the plant hormones abscisic acid (ABA) and gibberellin (GA) have been regarded as major regulators of 


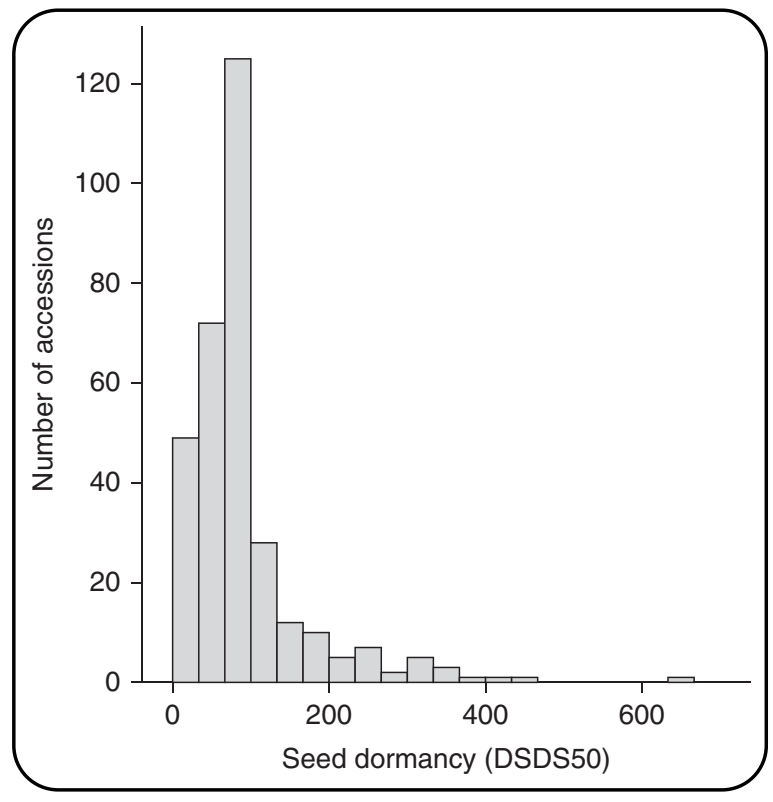

Figure 2 Dormancy in a worldwide population. Dormancy levels in a collection of natural accessions of Arabidopsis. Dormancy is expressed as an after-ripening requirement (the number of days of seed dry storage required to reach $50 \%$ of germination; DSDS50).

seed dormancy. ABA promotes dormancy and GA stimulates germination. Low levels of ABA during seed development have been shown to reduce dormancy, whereas enhanced expression of ABA biosynthesis genes is associated with increased ABA levels and enhanced dormancy (Nambara and Marion-Poll, 2003). During imbibition of seeds, the ABA level decreases in both dormant and non-dormant seeds. However, the decrease is stronger in nondormant seeds and ABA levels increase again after a few days in dormant seeds (Ali-Rachedi et al., 2004). The essential role of GA in germination has been demonstrated by the inability of GA biosynthesis mutants to germinate (Koornneef and van der Veen, 1980). This block of germination is caused by the DELLA growth inhibitors that restrain plant growth and as such inhibit the radicle from breaking through the endosperm and seed coat. GA overcomes this DELLA-mediated growth restraint. Other key factors in GA signalling are the GA receptors gibberellin insensitive dwarf1 and 2 and the F-box proteins SLEEPY1 and SNEEZY (Daviere and Achard, 2013). It is the intrinsic balance between ABA and GA in the seeds, over the actual amounts, that is thought to affect the dormancy state (Finch-Savage and Leubner-Metzger, 2006). Recently, other hormones have been proposed to also affect seed dormancy, including auxin, brassinosteriods (BR) ethylene and strigolactones, often acting in cross talk (Arc et al., 2013; Corbineau et al., 2014). See also: Abscisic Acid (ABA); Gibberellin - Mechanism of Action; Strigolactones: A New Class of Plant Hormones with Multifaceted Roles

\section{Seed after-ripening}

Primary dormancy is overcome by seed dry storage, also referred to as after-ripening. As a result of after-ripening, the window of

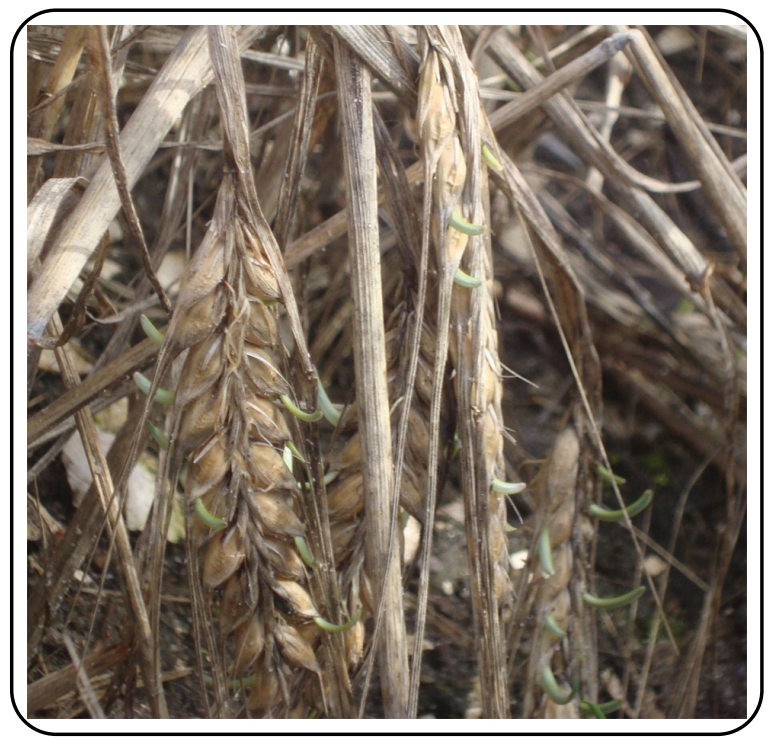

Figure 3 Preharvest sprouting in a barley field in the Netherlands observed on 1 September 2010. Seeds attached to their mother plant have germinated due to a combination of low dormancy and high humidity.

environmental conditions that allow seed germination widens. At the physiological level there is quite some knowledge on seed after-ripening; however, not much is known about the molecular mechanisms that control this process. The rate of after-ripening varies depending on environmental conditions. It slows down under low humidity and low oxygen. Transcriptome and proteome changes that have been reported during seed after-ripening still require a certain humidity. Under these conditions, it is probably reactive oxygen species (ROS) that affect after-ripening. ROS are mostly known for their deleterious effects and they can lead to protein carbonylation, an irreversible oxidation that causes the loss of protein function. This carbonylation is not necessarily harmful, as seen by the fact that it has been associated with the completion of germination (Job et al., 2005; Oracz et al., 2007). Moreover, it is proposed that the oxidation of dormancy-specific mRNAs leads to the release of dormancy by after-ripening, by avoiding their translation upon seed imbibition (Bazin et al., 2011). See also: Oxidative Stress and Redox Signalling in Plants

\section{Transcription and translation}

Numerous transcriptome studies have been performed to identify genes that are involved in the induction, maintenance and release of seed dormancy. Where, at first, these were mostly based on direct comparisons between different dormancy stages, later they contained temporal and spatial detail during seed development, dormancy release, cycling and germination (Figure 4). As a next step, these data were taken together to build networks that can help identify master regulators. One such an attempt is SeedNet, which highlights interactions between known regulators of dormancy and germination (Bassel et al., 2011). Since gene functioning is determined by proteins also, the proteome has been 


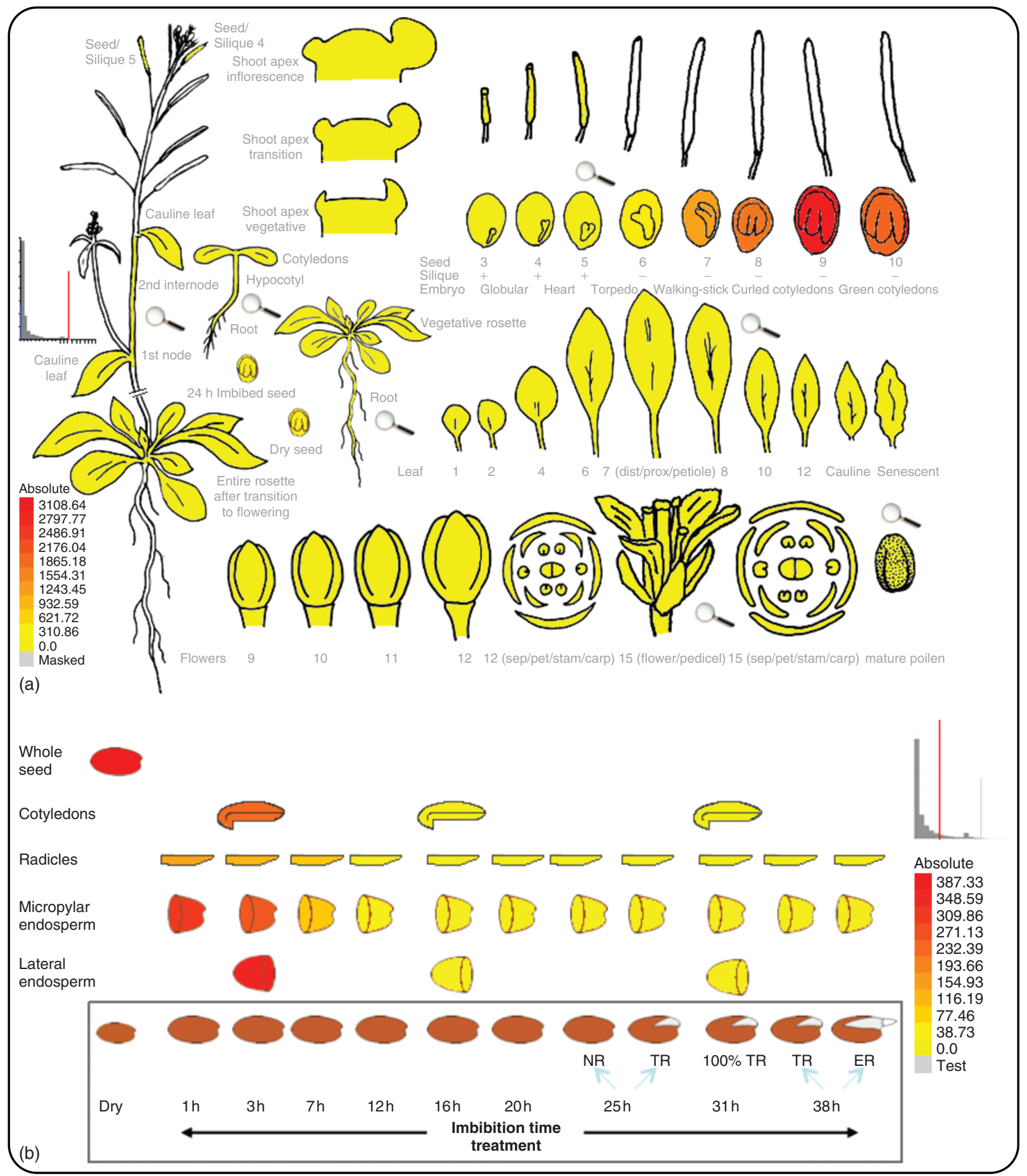

Figure 4 Schematic representation of the expression of the DELAY OF GERMINATION 1 (DOG1) gene in different plant tissues. (a) DOG1 expression is presented on the Arabidopsis developmental map. Data retrieved from the Arabidopsis eFP browser at barutoronto.ca (Winter et al., 2007). (b) DOG1 expression in presented on the Arabidopsis time series microarrays. Data retrieved from the Wageningen Seed Lab eFP Browser at www.wageningenseedlab.nl/resources (Dekkers et al., 2013). 
extensively analysed in relation to seed dormancy. This revealed that germination in dormant seeds is not blocked by the inhibition of protein synthesis (Chibani et al., 2006).

\section{Epigenetic regulation}

Epigenetics refers to heritable changes in gene expression (active versus inactive genes) that do not involve changes to the underlying DNA (deoxyribonucleic acid) sequence but that are caused by alterations in the chromatin structure. Several epigenetic marks have been related to dormancy maintenance and release. Among these is the trimethylation of histone $\mathrm{H} 3$ lysine 4 (H3K4me3) and lysine 27. H3K4me3 is an 'activating mark' leading to transcription that is more prone in nondormant seeds, while transcription is inhibited by $\mathrm{H} 3 \mathrm{~K} 27 \mathrm{me} 3$ that is more often seen in dormant seeds (Muller et al., 2012; Footitt et al., 2015). These epigenetic marks have also shown to vary accordingly during dormancy cycling (Footitt et al., 2015).

Other epigenetic regulators that are strikingly upregulated during the induction of seed dormancy are HISTONE MONOUBIQUITINATION 1 (HUB1) and REDUCED DORMANCY 2 (RDO2)/TRANSCRIPTION ELONGATION FACTOR $S$-II (TFIIS). HUB1 is involved in the monoubiquitination of histone $\mathrm{H} 2 \mathrm{~B}$; mutants in $H U B 1$ have reduced dormancy (Liu et al., 2007). RDO2 is required for seed dormancy, presumably by counteracting negative effects of increased chromatin compaction during seed maturation (Liu et al., 2011).

The acetylation of histones by histone acetyltransferases (HATs) and histone deacetylases (HDACs) has also been related with seed dormancy. Generally, acetylated histones are linked to an open chromatin configuration and, therefore, transcriptionally active loci, whereas deacetylation is associated with condensed chromatin structures and gene repression. Mutations in genes involved in histone deacetylation, like SWI-INDEPENDENT 3 LIKE 1 (SNL1), SNL2 and HISTONE DEACETYLASE 9 have increased acetylation levels and reduced seed dormancy (Wang et al., 2013; van Zanten et al., 2014). See also: Epigenetic Regulation in Plants

\section{Specific regulators}

The above-described regulatory pathways are important for seed dormancy, but they are not completely specific and can also influence other plant traits. For instance, many mutants in ABA/GA pathways or ROS signalling with a dormancy phenotype show altered plant growth or stress responses and mutations in the epigenetic regulator $H U B 1$ also influence flowering time (Cao et al., 2008). In this paragraph, we discuss a number of genes that are specific for seed dormancy. These genes are only expressed in seeds (Figure 4) and their mutants alter seed dormancy without affecting other plant traits.

The DOG1 gene is a key regulator of seed dormancy that was first identified in Arabidopsis. DOG1 is a major factor controlling natural variation and $\operatorname{dog} 1$ loss-of-function mutants are completely nondormant (Bentsink et al., 2006). The amount of DOG1 protein in freshly harvested seeds correlates with their dormancy level; and DOG1 slowly loses its function during seed storage, suggesting that it acts as a timer for seed dormancy release (Nakabayashi et al., 2012). DOG1 is a temperature sensor and low temperatures during seed maturation enhance DOG1 levels (Kendall et al., 2011; Nakabayashi et al., 2012). Homologues of DOG1 have been identified in various plant species. For some of these it has been shown that they are functionally conserved and enhance seed dormancy in species like Lepidium sativum (Graeber et al., 2014) and Triticum aestivum (Ashikawa et al., 2014). The molecular function of DOG1 is still unknown, but it has been shown to regulate dormancy largely independent from ABA, although both factors are likely to have common downstream targets (Nakabayashi et al., 2012).

Additional recently identified genes that are specific for dormancy and for which no clear relation with known pathways have been identified are REDUCED DORMANCY 5 (RDO5), MOTHER OF FT and TFL1 (MFT) and Sdr4. RDO5 has been described in Arabidopsis and its mutant seeds show strongly reduced dormancy (Xiang et al., 2014). RDO5 is a member of the PP2C phosphatases that lacks phosphatase activity. Natural variation in its sequence contributes to dormancy variation between Arabidopsis accessions (Amiguet-Vercher et al., 2015). RDO5 has an influence on dormancy similar to that of DOG1 and also seems to function largely independent of ABA.

MFT promotes dormancy in wheat (Nakamura et al., 2011) and Arabidopsis (Vaistij et al., 2013). The MFT gene underlies a germination QTL in wheat. It has a seed-specific expression pattern and is upregulated by low temperatures (Nakamura et al., 2011). MFT encodes a member of the plant phosphatidyl ethanolamine-binding protein (PEBP) family. Its precise mode of action is not known, but it has been shown to establish a negative feedback role in ABA signalling (Xi et al., 2010). Cloning of the rice dormancy QTL $S d r 4$ identified a gene encoding a protein with unknown function (Sugimoto et al., 2010). Sdr4 is preferentially expressed in seed tissues and positively regulates transcript levels of DOG1-like genes (Sugimoto et al., 2010).

All these four specific regulators have several characteristics in common. They are positive regulators of seed dormancy with a seed-specific expression. In addition, haplotype variation for these genes has been related with natural variation for seed dormancy. Interestingly, DOG1 and MFT transcript levels also showed a strong correlation with dormancy levels in Arabidopsis seeds that were stored in the soil under natural conditions (Footitt et al., 2011). The molecular mechanisms by which these regulators control seed dormancy and their relation with the ABA pathway are still poorly understood. Ongoing research should lead to a better understanding of these dormancy genes in the near future and contribute to reveal the similarities and dissimilarities in regulation between seed, bud, and other forms of dormancy.

\section{References}

Ali-Rachedi S, Bouinot D, Wagner MH, et al. (2004) Changes in endogenous abscisic acid levels during dormancy release and maintenance of mature seeds: studies with the Cape Verde Islands ecotype, the dormant model of Arabidopsis thaliana. Planta 219: 479-488.

Alonso-Blanco C, Bentsink L, Hanhart CJ, Blankestijn-de Vries $\mathrm{H}$ and Koornneef M (2003) Analysis of natural allelic variation 
at seed dormancy loci of Arabidopsis thaliana. Genetics 164: 711-729.

Amiguet-Vercher A, Santuari L, Gonzalez-Guzman M, et al. (2015) The IBO germination quantitative trait locus encodes a phosphatase 2C-related variant with a nonsynonymous amino acid change that interferes with abscisic acid signaling. New Phytologist 205: 1076-1082.

Ashikawa I, Mori M, Nakamura S and Abe F (2014) A transgenic approach to controlling wheat seed dormancy level by using Triticeae DOG1-like genes. Transgenic Research 23: 621-629.

Arc E, Sechet J, Corbineau F, Rajjou L and Marion-Poll A (2013) ABA crosstalk with ethylene and nitric oxide in seed dormancy and germination. Frontiers in Plant Science 4: 63.

Bassel GW, Lan H, Glaab E, et al. (2011) Genome-wide network model capturing seed germination reveals coordinated regulation of plant cellular phase transitions. Proceedings of the National Academy of Sciences of the United States of America 108: 9709-9714.

Baskin CC and Baskin JM (2014) Seeds: Ecology, Biogeography and Evolution of Dormancy and Germination. San Diego: Academic Press.

Bazin J, Langlade N, Vincourt P, et al. (2011) Targeted mRNA oxidation regulates sunflower seed dormancy alleviation during dry after-ripening. Plant Cell 23: 2196-2208.

Bentsink L, Jowett J, Hanhart CJ and Koornneef M (2006) Cloning of DOG1, a quantitative trait locus controlling seed dormancy in Arabidopsis. Proceedings of the National Academy of Sciences of the United States of America 103: 17042-17047.

Cao Y, Dai Y, Cui S and Ma L (2008) Histone H2B monoubiquitination in the chromatin of FLOWERING LOCUS C regulates flowering time in Arabidopsis. Plant Cell 20: 2586-2602.

Chibani K, Ali-Rachedi S, Job C, et al. (2006) Proteomic analysis of seed dormancy in Arabidopsis. Plant Physiology 142: 1493-1510.

Corbineau F, Xia Q, Bailly C and El-Maarouf-Bouteau H (2014) Ethylene, a key factor in the regulation of seed dormancy. Frontiers in Plant Science 5: 539.

Daviere JM and Achard P (2013) Gibberellin signaling in plants. Development 140: 1147-1151.

Debieu M, Tang C, Stich B, et al. (2013) Co-variation between seed dormancy, growth rate and flowering time changes with latitude in Arabidopsis thaliana. PLoS One 8: e61075.

Dekkers BJ, Pearce S, van Bolderen-Veldkamp RP, et al. (2013) Transcriptional dynamics of two seed compartments with opposing roles in Arabidopsis seed germination. Plant Physiology 163: 205-215.

Finch-Savage WE and Leubner-Metzger G (2006) Seed dormancy and the control of germination. New Phytologist 171: 501-523.

Footitt S, Douterelo-Soler I, Clay H and Finch-Savage WE (2011) Dormancy cycling in Arabidopsis seeds is controlled by seasonally distinct hormone-signaling pathways. Proceedings of the National Academy of Sciences of the United States of America 108: 20236-20241.

Footitt S, Muller K, Kermode AR and Finch-Savage WE (2015) Seed dormancy cycling in Arabidopsis: chromatin remodelling and regulation of $D O G 1$ in response to seasonal environmental signals. Plant Journal 81: 413-425.

Graeber K, Linkies A, Steinbrecher T, et al. (2014) DELAY OF GERMINATION 1 mediates a conserved coat-dormancy mechanism for the temperature- and gibberellin-dependent control of seed germination. Proceedings of the National Academy of Sciences of the United States of America 111: E3571-E3580.
Hilhorst HWM (1998) Seed dormancy. Seed Science Research 7: 221-223.

Job C, Rajjou L, Lovigny Y, Belghazi M and Job D (2005) Patterns of protein oxidation in Arabidopsis seeds and during germination. Plant Physiology 138: 790-802.

Kendall SL, Hellwege A, Marriot P, et al. (2011) Induction of dormancy in Arabidopsis summer annuals requires parallel regulation of DOG1 and hormone metabolism by low temperature and CBF transcription factors. Plant Cell 23: 2568-2580.

Kendall S and Penfield S (2012) Maternal and zygotic temperature signalling in the control of seed dormancy and germination. Seed Science Research 22: S23-S29.

Koornneef M and van der Veen JH (1980) Induction and analysis of gibberellin sensitive mutants in Arabidopsis thaliana (L.) Heynh. Theoretical and Applied Genetics 58: 257-263.

Lin SY, Sasaki T and Yano M (1998) Mapping quantitative trait loci controlling seed dormancy and heading date in rice, Oryza sativa L., using backcross lines. Theoretical and Applied Genetics 96: 997-1003.

Liu YX, Koornneef M and Soppe WJJ (2007) The absence of histone H2B monoubiquitination in the Arabidopsis hubl (rdo4) mutant reveals a role for chromatin remodeling in seed dormancy. Plant Cell 19: 433-444.

Liu YX, Geyer R, van Zanten M, et al. (2011) Identification of the Arabidopsis REDUCED DORMANCY 2 Gene Uncovers a Role for the Polymerase Associated Factor 1 Complex in Seed Dormancy. PLoS One 6: e22241.

Muller K, Bouyer D, Schnittger A and Kermode AR (2012) Evolutionarily conserved histone methylation dynamics during seed life-cycle transitions. PLoS One 7: e51532.

Nakabayashi K, Bartsch M, Xiang Y, et al. (2012) The time required for dormancy release in Arabidopsis is determined by DELAY OF GERMINATION 1 protein levels in freshly harvested seeds. Plant Cell 24: 2826-2838.

Nakamura S, Abe F, Kawahigashi H, et al. (2011) A wheat homolog of MOTHER OF FT AND TFL1 acts in the regulation of germination. Plant Cell 23: 3215-3229.

Nambara E and Marion-Poll A (2003) ABA action and interactions in seeds. Trends in Plant Science 8: 213-217.

Oracz K, El-Maarouf Bouteau H, Farrant JM, et al. (2007) ROS production and protein oxidation as a novel mechanism for seed dormancy alleviation. Plant Journal 50: 452-465.

Rodríguez M, Barrero JM, Corbineau F, Gubler $\mathrm{F}$ and Benech-Arnold RL (2015) Dormancy in cereals (not too much, not so little): about the mechanisms behind this trait. Seed Science Research 25: 99-119.

Sugimoto K, Takeuchi Y, Ebana K, et al. (2010) Molecular cloning of $S d r 4$, a regulator involved in seed dormancy and domestication of rice. Proceedings of the National Academy of Sciences of the United States of America 107: 5792-5797.

Vaistij FE, Gan Y, Penfield S, et al. (2013) Differential control of seed primary dormancy in Arabidopsis ecotypes by the transcription factor SPATULA. Proceedings of the National Academy of Sciences of the United States of America 110: 10866-10871.

Wang Z, Cao H, Sun Y, et al. (2013) Arabidopsis paired amphipathic helix proteins SNL1 and SNL2 redundantly regulate primary seed dormancy via abscisic acid-ethylene antagonism mediated by histone deacetylation. Plant Cell 25: 149-166. 
Winter D, Vinegar B, Nahal H, et al. (2007) An "Electronic Fluorescent Pictograph" browser for exploring and analyzing large-scale biological data sets. PLoS One 2: e718.

Xi W, Liu C, Hou X and Yu H (2010) MOTHER OF FT AND TFL1 regulates seed germination through a negative feedback loop modulating ABA signaling in Arabidopsis. Plant Cell 22: 1733-1748.

Xiang Y, Nakabayashi K, Ding J, et al. (2014) REDUCED DORMANCY 5 encodes a protein phosphatase $2 \mathrm{C}$ that is required for seed dormancy in Arabidopsis. Plant Cell 26: 4362-4375.

van Zanten M, Zoll C, Wang Z, et al. (2014) HISTONE DEACETYLASE 9 represses seedling traits in Arabidopsis thaliana dry seeds. Plant Journal 80: 475-488.

\section{Further Reading}

Cooke JEK, Eriksson ME and Junttila O (2012) The dynamic nature of bud dormancy in trees: environmental control and molecular mechanisms. Plant, Cell \& Environment 35: 1707-1728.

Gazzarrini S and Tsai AYL (2015) Hormone cross talk during seed germination. Essays in Biochemistry 58: 151-164.

Graeber K, Nakabayashi K, Miatton E, Leubner-Metzger G and Soppe WJJ (2012) Molecular mechanisms of seed dormancy. Plant, Cell \& Environment 35: 1769-1786.

Nonogaki H (2014) Seed dormancy and germination - emerging mechanisms and new hypotheses. Frontiers in Plant Science 5: 233. 